\title{
Treatment of early accelerated AMR with eculizumab in antibody incompatible renal transplantation
}

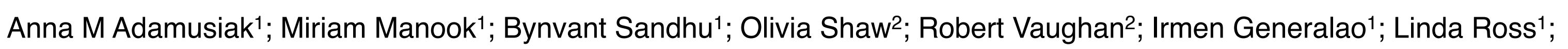
Professor Anthony Dorling ${ }^{1}$; Nicos Kessaris ${ }^{1}$; Professor Nizam Mamode ${ }^{1}$

1 - Department of Renal Transplantation at Guy's Hospital in London

2 - Clinical Transplantation Laboratory at at Guy's Hospital in London

\section{Introduction}

Early accelerated antibody-mediated rejection (AMR) within 2 weeks after antibody-incompatible renal transplantation can be defined as sudden deterioration in graft function with oligoanuria that requires re-commencing on dialysis, does not improve with pheresis and often results in graft loss. The most common mechanism of AMR is binding of antibodies to antigens on endothelial cells in the kidney followed by complement activation. Eculizumab is a monoclonal antibody that specifically targets and blocks action of complement. It can be used as a salvage therapy in AMR refractory to standard treatment.

\section{Methods}

Over a period of 6 years 10 patients who underwent antibody incompatible living donor renal transplantation were treated with eculizumab. 4 of them were blood group (ABOi) and 6 were HLA-antibody incompatible ( $\mathrm{HLAi})$. The initial protocol was 5 doses on weekly bases. One of the patients had a 9 weeks course and one a 4 weekly course. All patient were receiving standard AMR treatment including antibody removal with no improvement in renal function.

\section{Results}

Mean age of patients treated was $43 \pm 17$ years (ranged $18-66$ ) and 9 of 10 were female. Mean baseline donor specific antibody (DSA) level for HLAi patients was 49280 $\pm 21230 \mathrm{MFI}$ (ranged 26526 - 84022). 2 patients lost their grafts and underwent nephrectomy (both $\mathrm{ABOi}$ ) and 2 patients died with no evidence of graft recovery (both $\mathrm{HLAi}$ ) before finishing a scheduled treatment with eculizumab. Follow-up of patients ranged between 2 months and 2 years. Mean creatinine on the last visit was $133 \pm 53 \mathrm{umol} / \mathrm{L}$.

\section{Results cont.}

Efficacy of eculizumab in our cohort was $60 \%$. Relative risk of treatment failure with standard AMR treatment versus salvage therapy with eulizumab was $2.5(95 \% \mathrm{Cl} 1.17-5.34) \mathrm{p}=0.02$; number needed to harm $=1.67(3.37-1.11)$. There was no statistically significant difference in either baseline DSA pre transplant or highest level of DSA post transplant between patients whose graft function did and did not recover. Mean DSA value on the last follow up was $14710 \pm 11342 \mathrm{MFI}$ (ranged 3251 $-26218)$.

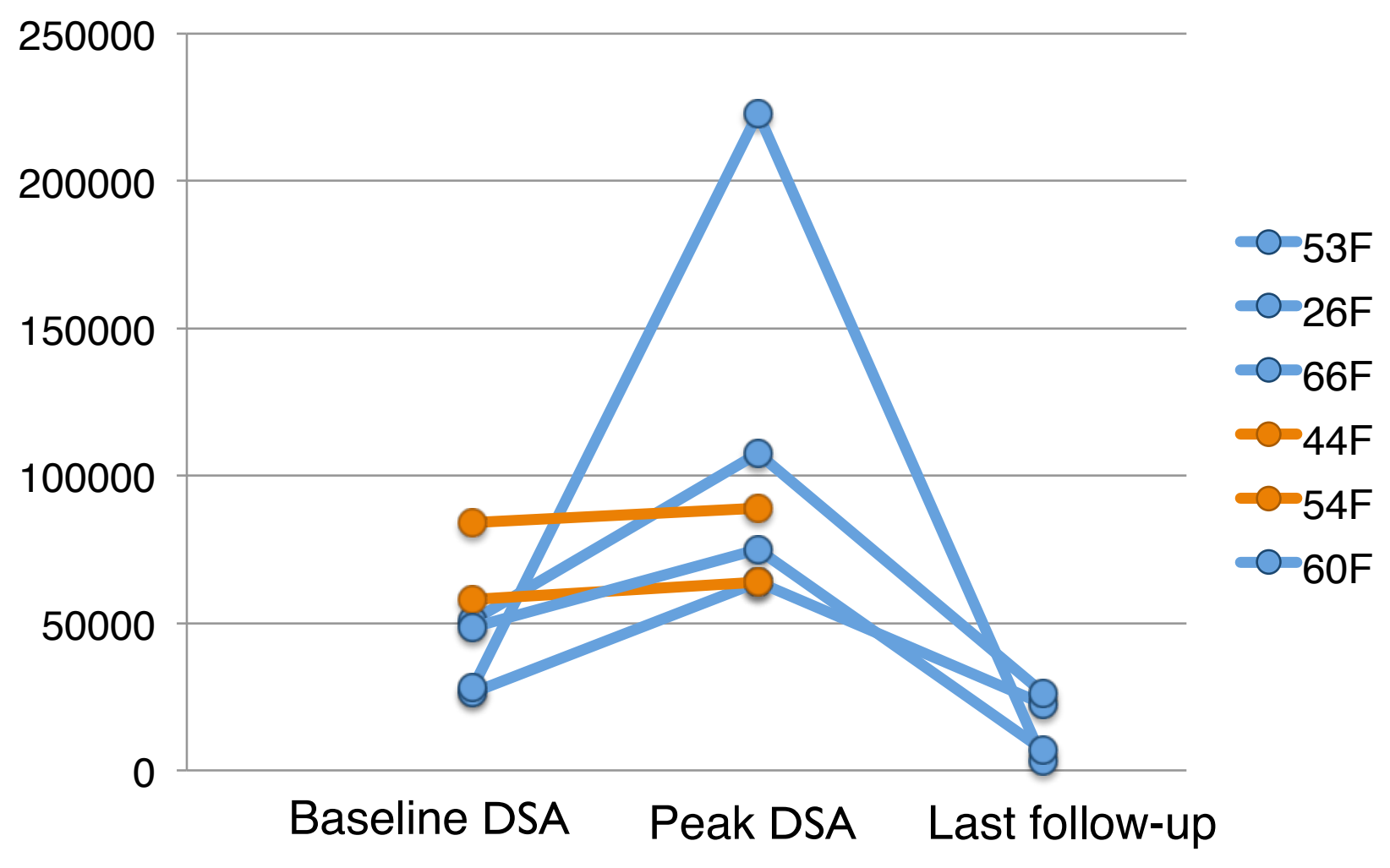

Figure 1. Comparison of DSA levels between patients whose graft recovered (blue) and patients whose graft did not recover (orange). The latter died during the eculizumab rescue therapy. DSA value given in Mean Fluorescence Intensity (MFI).

\section{Discussion}

Eculizumab can be successful in treatment of accelerated antibody mediated rejection. Multicentre analysis of treatment outcomes may allow better identification of patients who would likely benefit from short course of eculizumab as a salvage therapy.

\section{References}

Difference in outcomes after antibody-mediated rejection between abo-incompatible and positive cross-match transplantations. Couzi L, Manook M, Perera R, Shaw O, Ahmed Z, Kessaris N, Dorling A, Mamode N. Transpl Int. 2015 Oct;28(10):1205-15.

The use of eculizumab in renal transplantation. Barnett AN, Asgari E, Chowdhury P, Sacks SH, Dorling A, Mamode N. Clin Transplant. 2013 May-Jun;27(3):E216-29.

\section{Guy's and St Thomas' WHS NHS Foundation Trust}

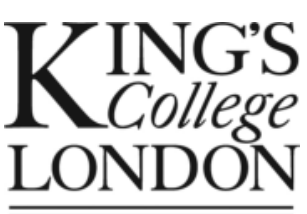

www.kcl.ac.uk

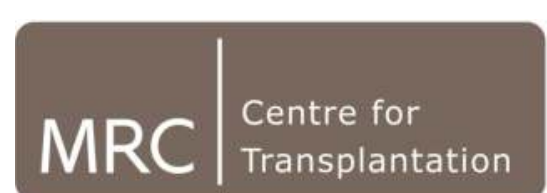

$\begin{array}{lll}1 & 1 \\ 1 & 1\end{array}$

4I II I iniliti KING'S

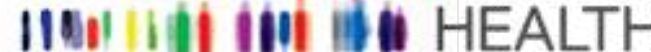
i i is in bi ilili iloi PARTNERS 